\title{
Converting Okara to Superabsorbent Hydrogel as Soil Supplement for Enhancing Growth of Choy Sum (Brassica sp.) under Water-Limited Conditions
}

Jingling Zhu ${ }^{\dagger}{ }^{\dagger}, 1$ Wee Kee Tan,${ }^{\dagger, 1}$ Xia Song, ${ }^{\dagger}$ Zhengyang Gao, ${ }^{\dagger}$ Yuting Wen, ${ }^{\star}$ Choon Nam Ong, ${ }^{\S}$ Chiang Shiong Loh, ${ }^{\#}$ Sanjay Swarup, ${ }^{\#}$ and Jun Li ${ }^{\ddagger *}$

${ }^{\dagger}$ NUS Environmental Research Institute, National University of Singapore, 5A Engineering Drive 1, Singapore 117411, Singapore

Department of Biomedical Engineering, Faculty of Engineering, National University of Singapore, 7 Engineering Drive 1, Singapore 117574, Singapore

${ }^{\S}$ Saw Swee Hock School of Public Health, National University of Singapore, 12, Science Drive 2, Singapore 117549, Singapore

\# Department of Biological Sciences, National University of Singapore, 14 Science Drive 4, Singapore 117543, Singapore

* Corresponding author.

E-mail: jun-li@nus.edu.sg; jun-li@u.nus.edu (J. Li)

${ }^{1}$ Note: Jingling Zhu and Wee Kee Tan contributed equally to this work.

\section{SUPPORTING INFORMATION}

Manuscript ID: sc-2020-02181f

5 pages, 4 figures

\section{Table of Contents}



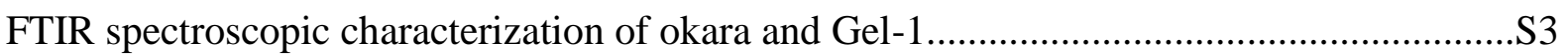

Water holding and water retention of potting mix supplemented with gels.........................S3

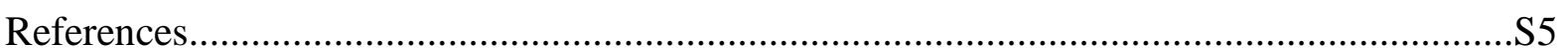


HPLC determination of monomer conversion
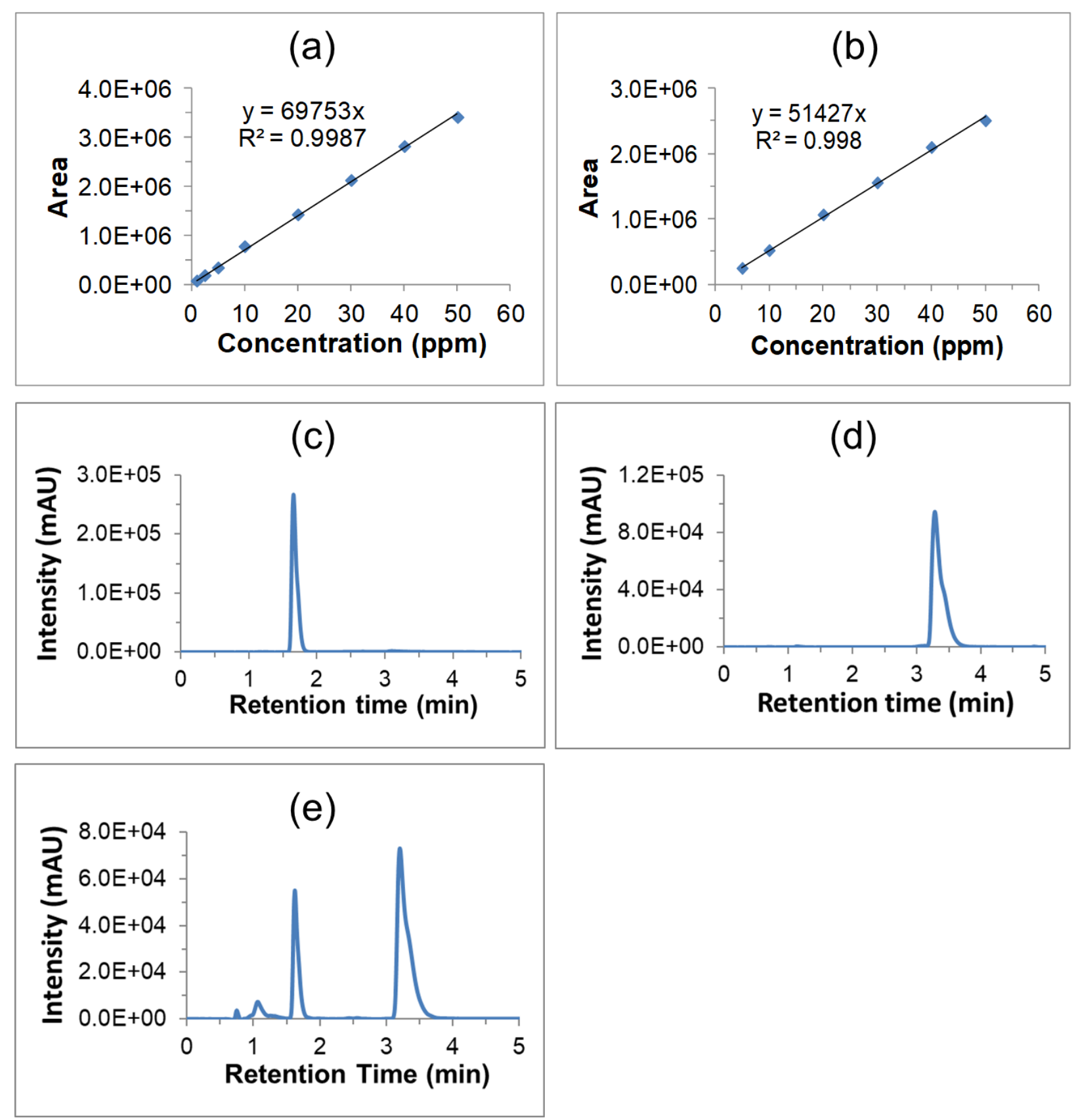

Figure S1. Calibration curves for (a) AAm over the range of 1-50 ppm, and (b) AA over the range of 5-50 ppm. UV-vis absorbance at $200 \mathrm{~nm}$ was used for quantification. Representative HPLC chromatogram of (c) AAm at $1.65 \mathrm{~min}$, (d) AA at $3.27 \mathrm{~min}$, and (e) AAm and AA residuals in Gel-1. 


\section{FTIR spectroscopic characterization of okara and Gel-1}

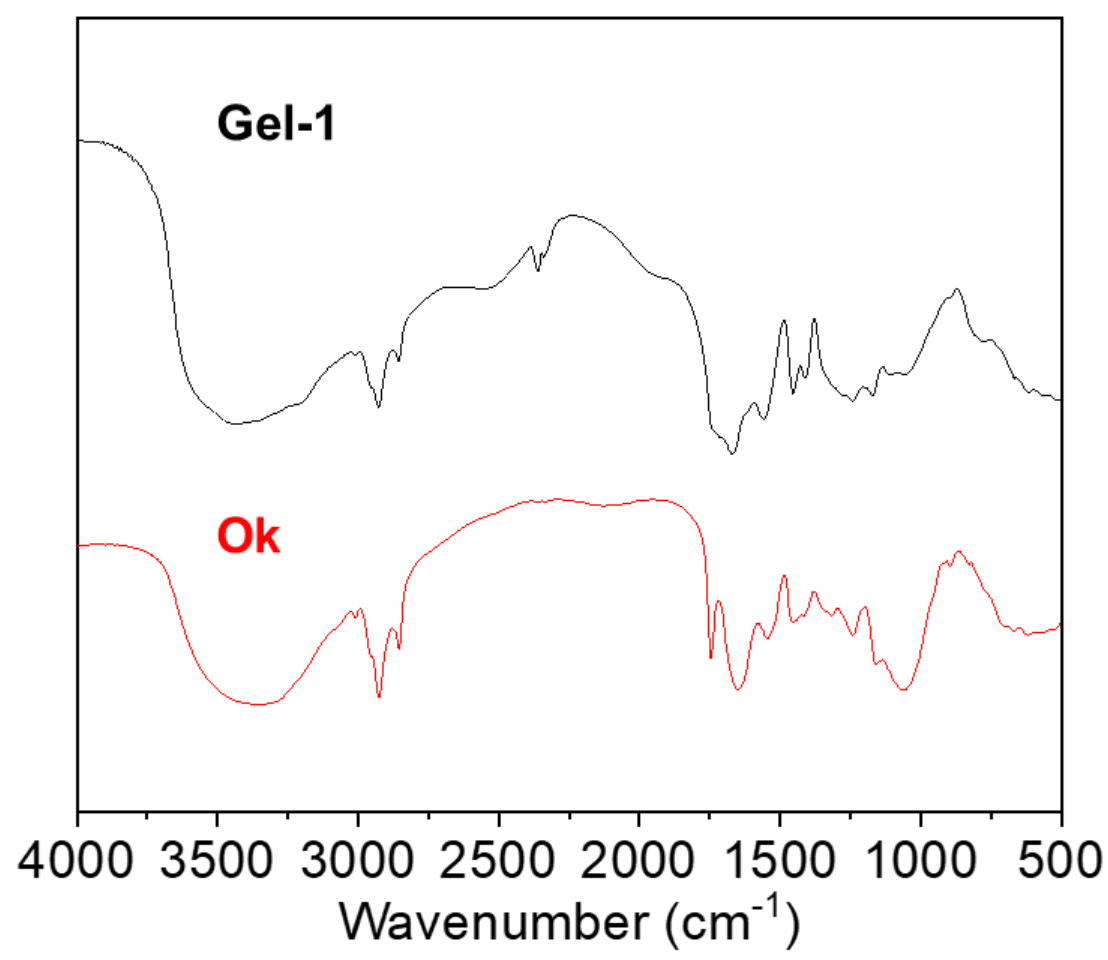

Figure S2. FTIR spectra of Gel-1 and Ok in the form of KBr discs.

\section{Water holding and water retention of potting mix supplemented with gels}

As we aim to apply the gel powders (small size) as soil supplement, the water holding and retention capacities of potting mix supplemented with different contents of gel powders were studied. A study on potting mix containing $0.5-5 \%$ (w/w) of Gel-1, Gel-2, or Gel-3 was carried out. As shown in Figures S3 and S4, the water holding and retention capacities of the potting mix increased with increasing content in the gel powders. The trend of the three gels was the same and there was no significant difference between the three gels for promoting the water holding and retention capacities. It is noted that Gel-3 has lower water absorbency capacity than the other two gels, but its water holding and retention capacities are comparable to Gel-1 and Gel-2. It is attributed to the higher AAm content in Gel-3. AAm is reported to have better salt-resistance than AA, ${ }^{1,2}$ which improved Gel-3's water holding, and retention properties in nutrient and salt rich potting mix. 


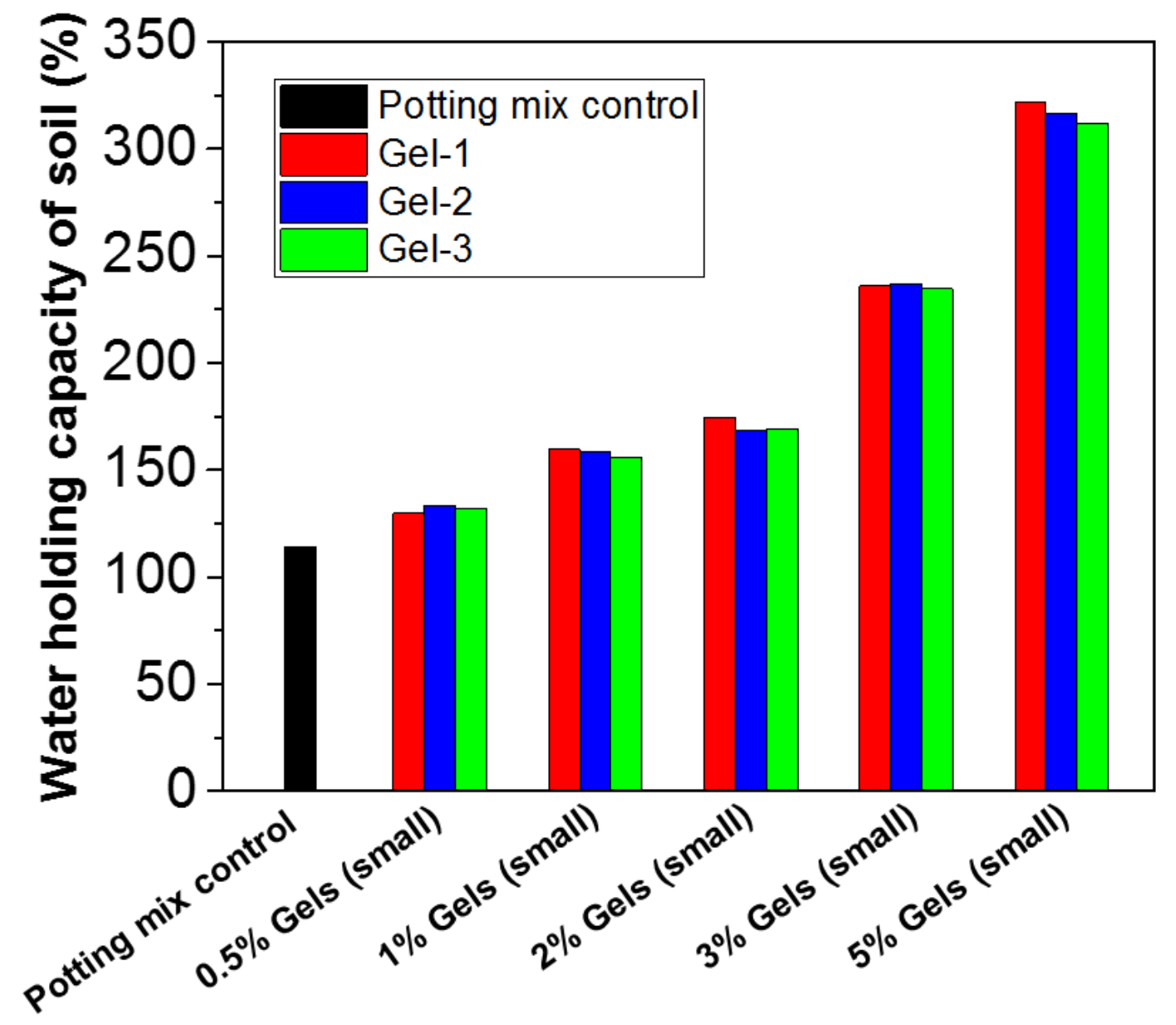

Figure S3. Water holding measurements of potting mix supplemented with $0.5 \%, 1 \%, 2 \%$, $3 \%$, and $5 \%$ gel powders (small size). Commercial potting mix (used as received) was used as control. 

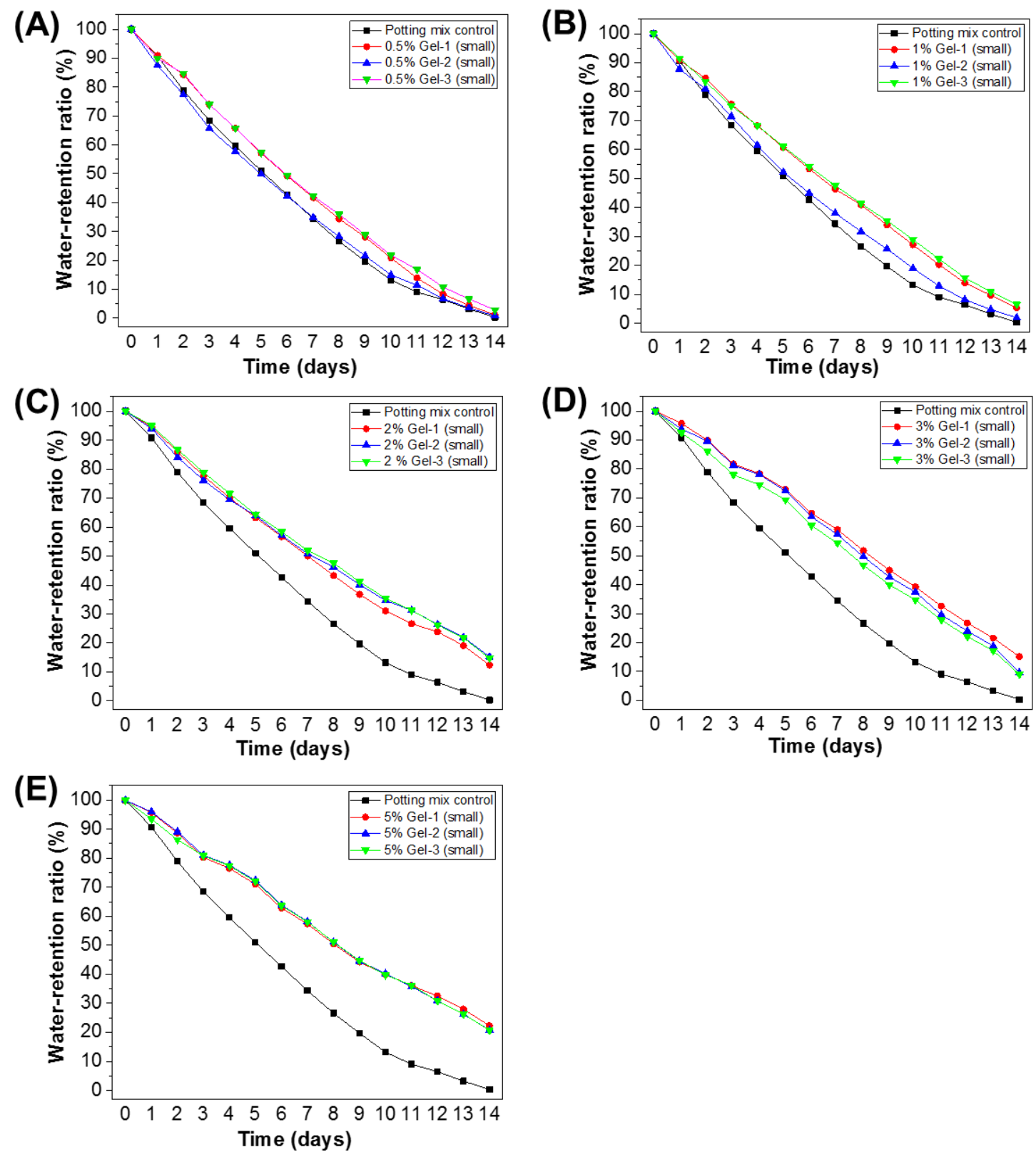

Figure S4. Water retention measurements of potting mix supplemented with (A) $0.5 \%$, (B) $1 \%$, (C) $2 \%$, (D) $3 \%$, and (E) $5 \%$ of gel powders (small size). Commercial potting mix (use as received) was used as control.

\section{References}

(1) Zhang, Y.; Wang, L.; Li, X.; He, P., Salt-resistant superabsorbents from inverse-suspension polymerization of PEG methacrylate, acryamide and partially neutralized acrylic acid. $J$. Polym. Res. 2011, 18 (2), 157-161.

(2) Tomar, R. S.; Gupta, I.; Singhal, R.; Nagpal, A. K., Synthesis of poly(acrylamide-co-acrylic acid) based superabsorbent hydrogels: study of network parameters and swelling behaviour. Polym-plast. Technol. 2007, 46 (5), 481-488. 